\title{
Rainfall measurement from the opportunistic use of an Earth-space link in the Ku band
}

\author{
L. Barthès and C. Mallet \\ Université de Versailles Saint-Quentin, UMR8190 - CNRS/INSU, LATMOS-IPSL, Laboratoire Atmosphères Milieux, \\ Observations Spatiales, Quartier des Garennes, 11 Boulevard d'Alembert, 78280 Guyancourt, France
}

Correspondence to: L. Barthès (laurent.barthes@latmos.ipsl.fr)

Received: 28 November 2012 - Published in Atmos. Meas. Tech. Discuss.: 26 February 2013

Revised: 3 June 2013 - Accepted: 16 July 2013 - Published: 29 August 2013

\begin{abstract}
The present study deals with the development of a low-cost microwave device devoted to the measurement of average rain rates observed along Earth-satellite links, the latter being characterized by a tropospheric path length of a few kilometres. The ground-based power measurements, which are made using the Ku-band television transmissions from several different geostationary satellites, are based on the principle that the atmospheric attenuation produced by rain encountered along each transmission path can be used to determine the path-averaged rain rate. This kind of device could be very useful in hilly areas where radar data are not available or in urban areas where such devices could be directly placed in homes by using residential TV antenna.

The major difficulty encountered with this technique is that of retrieving rainfall characteristics in the presence of many other causes of received signal fluctuation, produced by atmospheric scintillation, variations in atmospheric composition (water vapour concentration, cloud water content) or satellite transmission parameters (variations in emitted power, satellite pointing). In order to conduct a feasibility study with such a device, a measurement campaign was carried out over a period of five months close to Paris.

The present paper proposes an algorithm based on an artificial neural network, used to identify dry and rainy periods and to model received signal variability resulting from effects not related to rain. When the altitude of the rain layer is taken into account, the rain attenuation can be inverted to obtain the path-averaged rain rate. The rainfall rates obtained from this process are compared with co-located rain gauges and radar measurements taken throughout the full duration of the campaign, and the most significant rainfall events are analysed.
\end{abstract}

\section{Introduction}

The accurate measurement of medium-scale rain intensity and the precise localization of precipitation are important tasks in the study of the water cycle, and also represent major components of the physics of climate. Moreover, the various issues arising from the variability of precipitation over time and space are not only scientific. Knowledge of rainfall variability in the short term (extreme events) and long term (management of water resources) can also be beneficial in terms of the avoidance of human and material damage caused by these phenomena. The present study investigates an inexpensive microwave system used to observe rain at a medium spatial resolution and a high temporal resolution.

The most commonly used sensors for rainfall measurements are weather radar, rain gauges, disdrometers and remote sensing satellites. Although the latter make it possible to monitor precipitation on a global scale, microwave sensors using current technology must be positioned in a low Earth orbit, when measurements are needed with a resolution of a few kilometres. The resulting observation frequency (for a single satellite) is approximately twice a day, which is very low when compared to the dynamics of rainfall events. Ground-based weather radar systems cover an area of approximately $30000 \mathrm{~km}^{2}$, and have a revisit frequency of the order of a few minutes and a spatial resolution of approximately $1 \mathrm{~km}$. The unavoidable costs and human resource requirements of such radars make their implementation possible only in some specific regions of Earth. Rain gauges allow for spot observations to be made with a variable time step, depending on the rainfall intensity, and only in the presence of a dense network of such sensors can the spatial variability 
of rainfall events be correctly observed. Furthermore, the deployment and maintenance of such networks can be relatively complex and expensive, especially in mountainous areas, dense forests, wetlands, etc.

By working with operational point-to-point microwave telecommunication links, Upton et al. (2005), Messer et al. (2006), Leijnse et al. (2007), Zinevich et al. (2009), Schleiss and Berne (2010), Kaufmann and Rieckermann (2011), Wang et al. (2012), Overeem et al. (2013) and Fenicia et al. (2012) have shown that the path-averaged rain rate can be estimated from attenuation measurements. However, ground-based microwave link attenuations are provided by local telecom operators, leading to many practical constraints (in particular, coarse precision due to quantization errors and low temporal resolution (typically $15 \mathrm{~min}$ )). Furthermore, this type of data tends to be available mainly in urban zones, but not in rural areas.

There are currently more than 200 geostationary satellites deployed by broadcast or telecommunication companies, transmitting relatively strong Ku-band (10.7-12.7 GHz) microwave signals towards Earth and covering the entire globe. Positioned on quasi-geostationary orbits, these satellites thus represent continuously available microwave sources, whose apparent positions remain relatively stable. When viewed from Earth, the apparent positions of these satellites nevertheless impose restrictions on the specific directions (and thus atmospheric locations) along which $\mathrm{Ku}-$ band rain attenuation measurements can be made.

In the $\mathrm{Ku}$ band, electromagnetic transmission can be strongly affected by rain attenuation together with other less significant but considerably more frequent effects, due to atmospheric gases (oxygen and water vapour) and non-precipitating water (cloud).

In order to observe rainfall events at an intermediate resolution, between that provided by radar and rain gauges, the opportunistic use of these microwave sources was investigated based on the use of a low-cost device requiring significantly less maintenance than a network of rain gauges. A passive ground-based microwave system, capable of estimating the average rain rate along the Earth-satellite link (hereafter referred to as a "Ku device"), was thus developed. Ground-based power measurements are achieved by receiving Ku-band signals from several operational geostationary satellites, as was done in the experiments described by $\mathrm{Ku}-$ mar et al. (2008), Maitra et al. (2007) and Ramachandran and Kumar (2004). The atmospheric attenuation along the Earthspace link are then used to derive the path-averaged rain rates. Table 1 summarizes the main characteristics of weather radars, rain gauge networks and the Ku device. The proposed device is as simple as possible; the final objective is in fact to define a measurement method that could be easily deployed by using existing dish antennas available everywhere in the world. In fact, in many parts of the globe, subjected to significant climatic risk, no operational radar network is available, while microwave systems for television reception are deployed for a long time. The present paper describes the method used to retrieve the path-averaged rain rate from $\mathrm{Ku}-$ band signals received from geostationary satellites. In an initial step, the measurement principle is described, and the expected accuracy of the Ku-band attenuation measurements is estimated. A time series of raindrop size distributions is used to estimate the Ku-band attenuation and corresponding rain rate in order to study the influence on attenuation of rain inhomogeneity and raindrop size distribution along the link path. This section thus provides a relationship between atmospheric attenuation and rain rate.

In a second step, the experimental device and the measurement campaign designed to test the feasibility of rainfall measurements using this device are described. Groundbased power measurements were carried out by receiving various Ku-band TV channels from different geostationary satellites. The experimental microwave system was installed close to Paris during the summer and autumn of 2010. Other co-located rain observations (rain radar, rain gauges) used for comparison are also presented.

The third step deals with proposed methods for the retrieval of rain rates from the received microwave signal, and quantification of the expected rain rate accuracy. In practice, the proposed device measures received power only; however, the reference level of the transmitted signal, relative to which the rain attenuation is computed, remains unknown. In the case of Earth-satellite microwave links, this problem is considerably more complex than in the case of point-to-point microwave links, because the measured signal strength depends on several factors, including not only the atmospheric attenuation resulting from a number of processes such as rain, snow, hail, graupel, water vapour concentration, cloud water content, turbulence and air temperature, but also the position of the satellite when viewed from the ground. Most TV satellites are not in fact perfectly geostationary, since they have quasi-circular (slightly elliptic) orbits, which do not lie exactly in the equatorial plane: these are so-called geosynchronous orbits which, during the day, induce small relative movements of the satellite with respect to Earth. These, in turn, lead to small changes in the nominal direction of the transmitted microwave beam, and therefore to small fluctuations in the received signal level (depending on the antenna aperture). In this section a method is proposed for the identification of dry and rainy periods, and for the estimation of the signal reference level during rainy periods. Finally, by taking the altitude of the rain into account, the estimated rain attenuation is retrieved, from which the path-averaged rain rate can be obtained. The proposed algorithm remains simple because only one channel is used, at a fixed polarization and frequency; more-sophisticated solutions could be developed in the future by making use of a greater number of channels, at different frequencies and polarizations.

In the last section of this paper, the derived rain rates are compared with co-located rain gauge and rain radar measurements. The statistics obtained from the full experimental 
Table 1. Main characteristics of weather radars, rain gauge networks and the Ku device.

\begin{tabular}{llllcc}
\hline & $\begin{array}{l}\text { Typical } \\
\text { temporal } \\
\text { resolution }\end{array}$ & $\begin{array}{l}\text { Typical } \\
\text { spatial } \\
\text { resolution }\end{array}$ & Area & $\begin{array}{c}\text { Cost } \\
\text { (purchase and } \\
\text { installation) }\end{array}$ & $\begin{array}{c}\text { Mainte- } \\
\text { nance }\end{array}$ \\
\hline Radar & $5 \mathrm{~min}$ & $1 \mathrm{~km}^{2}$ & $30000 \mathrm{~km}^{2}$ & $* * * *$ & $* * *$ \\
\hline $\begin{array}{l}\text { Rain gauge } \\
\text { network }\end{array}$ & $1 \mathrm{~min}-24 \mathrm{~h}$ & Spot & $\begin{array}{l}\text { Proportional } \\
\text { \#pluviometers }\end{array}$ & $* *$ & $* * *$ \\
\hline $\begin{array}{l}\text { KU device using } \\
\text { one satellite }\end{array}$ & $10 \mathrm{~s}$ & $5-6 \mathrm{~km}$ & $5-6 \mathrm{~km}$ & $*$ & $*$ \\
\hline $\begin{array}{l}\text { KU device using } \\
\text { all available } \\
\text { satellites }\end{array}$ & $10 \mathrm{~s}$ & $\sim 1 \mathrm{~km}^{2}$ & $\sim 20 \mathrm{~km}^{2}$ & $*$ & $*$ \\
\hline
\end{tabular}

period are presented, and, finally, the most significant rainfall events are analysed.

\section{Physical context}

Attenuation is the main factor influencing propagation over satellite microwave links. This phenomenon is due to the presence of several types of atmospheric component: gases, clouds and rain along the propagation path (Ulaby et al., 1981). Concerning temporal and spatial variability, each of these components has a different behaviour. In the case of gas-induced attenuation, the extinction coefficient is equal to the absorption coefficient (Liebe et al., 1993). In the troposphere, oxygen and water vapour are the only gases contributing significantly to absorption in the microwave spectrum. In the case of a satellite slant path, gaseous absorption can be considered to be homogeneous over the horizontal range of the link, and variations in gas-induced attenuation are caused mainly by variations in the quantity of water vapour encountered along the slant path. Gaseous attenuation never exceeds $0.3 \mathrm{~dB}$ at frequencies close to $10 \mathrm{GHz}$, and an elevation angle of $30^{\circ}$ (ITU-R, 2012a).

The interaction of electromagnetic waves with tropospheric water particles may involve both absorption and scattering, depending on the size of the particles relative to the wavelength of the transmitted beam. More precisely, the attenuation per unit volume depends on the density, shape, size distribution and dielectric properties of the particles contained in the volume. In the case of liquid water in clouds, scattering effects are negligible, and the Rayleigh approximation can thus be used for frequencies up to approximately $50 \mathrm{GHz}$ (Liebe et al., 1993). The resulting attenuation is proportional to the integrated liquid water content present along the link. As cloud coverage is characterized by substantial spatiotemporal variabilities, the corresponding liquid content and cloud-induced attenuation can vary strongly, although in absolute terms the attenuation remains below $1 \mathrm{~dB}$ at the fre- quencies and elevation angles considered in this study (ITU$\mathrm{R}, 2012 \mathrm{~b}$ ). In the case of ice particles, the absorption coefficient is much smaller than that of water, such that these particles do not play a significant role in the $\mathrm{Ku}$ band. Rain is thus the major contributor to Ku-band attenuation, and both absorption and scattering phenomena play an important role. The attenuation per unit volume depends on the extinction cross section of the water particles and their size distribution profile. The latter varies noticeably both in space and time, involving the specific dynamics of rain-induced attenuation, which are different to those induced by gases or clouds.

\subsection{Rain-atmospheric-attenuation relationship}

Considering scattering effects, the rain-specific attenuation $k_{f p \theta}(t, l)\left[\mathrm{dB} \mathrm{km}^{-1}\right]$ is related to the microphysical properties of rain at a given frequency $f$, polarization $p(p=H$, $p=V$ or $p=C$ for horizontal, vertical or circular polarization, respectively) and elevation angle $\theta$, described by the following expression:

$k_{f p \theta}(t, l)=4343 \int_{D_{\min }}^{D_{\max }} N(D, t, l) \sigma_{f p \theta}(D) \mathrm{d} D$,

where $N(D, t, l)\left[\mathrm{m}^{-4}\right]$ is the raindrop size distribution (DSD), i.e. the number of drops per cubic metre per unit increment of spherical equivolume drop diameter $D[\mathrm{~m}]$ (i.e. the diameter of a sphere with the same volume) present in the atmosphere at location $l$ and time $t$, and $\sigma_{f p \theta}(D)\left[\mathrm{m}^{2}\right]$ is the extinction cross section for a raindrop of diameter $D[\mathrm{~m}]$.

The rain attenuation $A_{f p \theta}^{\mathrm{Rain}}(t)[\mathrm{dB}]$ is thus obtained by integrating the specific attenuation along the slant path of length $L$ :

$A_{f p \theta}^{\mathrm{Rain}}(t)=\int_{0}^{L} k_{f p \theta}(t, l) \mathrm{d} l$. 
The extinction cross section $\sigma_{f p \theta}(D)$ of the raindrops depends on the transmitted frequency, the refractive index $n$ of the water, the size and shape of the raindrops, and the polarization and incidence angle of the electromagnetic wave. In this study, $\sigma_{f p \theta}(D)$ is considered to be independent of location $l$, even though temperature variations can lead to slight fluctuations in refractive index $n$ as the drop falls through the atmosphere. In the present study, a fixed temperature (set to $10^{\circ} \mathrm{C}$ ) and negligible induced errors in $\sigma_{f p \theta}(D)$ are assumed (Atlas and Ulbrich, 1977). Indeed, if the real temperature lies in the range $0-20^{\circ} \mathrm{C}$, whereas a fixed temperature of $10^{\circ} \mathrm{C}$ is assumed, the relative specific attenuation error at $12 \mathrm{GHz}$ is approximately $2 \%$ when the rain rate is equal to $10 \mathrm{~mm} \mathrm{~h}^{-1}$, and approximately $5.5 \%$ for a rain rate equal to $100 \mathrm{~mm} \mathrm{~h}^{-1}$. Under these conditions, the rain profile along the slant path of length $L$ can be treated as if it were a single layer of rain, with an equivalent drop size distribution $N_{\mathrm{e}}$ $(D, t)\left[\mathrm{m}^{-4}\right]$ defined by

$N_{\mathrm{e}}(D, t)=\frac{1}{L} \int_{0}^{L} N(D, t, l) \mathrm{d} l$.

The equivalent specific attenuation $k_{f p \theta}(t)$ can then be expressed as

$k_{f p \theta}(t)=4343 \int_{D_{\min }}^{D_{\max }} N_{\mathrm{e}}(D, t) \sigma_{f p \theta}(D) \mathrm{d} D$.

The simple relationship describing rain attenuation can be written as follows:

$A_{f p \theta}^{\mathrm{Rain}}(t)=k_{f p \theta}(t) L$.

In practice, although the DSD is generally unknown, by considering a gamma drop size distribution, the following empirical $k-R$ power law can be used to relate the specific attenuation to the rain rate:

$k_{f p \theta}(t)=a_{f p \theta} R^{b_{f p \theta}}(t)$,

This expression is shown to be an approximation, except in the low frequency and optical limits (Olsen et al., 1978). In the microwave domain, $a_{f p \theta}$ and $b_{f p \theta}$ depend on frequency and to a lesser extent on the drop size distribution, elevation angle and polarization.

\subsection{Accuracy of the $k-R$ power law relationship}

As shown above, the $k-R$ relation depends on the equivalent DSD features, which are the drop concentration and the shape of the DSD. These features depend on the atmospheric conditions (convective or stratiform rain for example) and their variability along the radio link $L$ (i.e. the spatial scale under consideration).
Initially, a homogeneous layer with different DSD concentrations and shapes is considered. As pointed out by Jameson (1991), the $k-R$ dependence on shape, for a homogeneous rain layer, is related to frequency. These authors show that for frequencies close to $25 \mathrm{GHz}$, this dependence is weak, whereas a stronger dispersion occurs at lower frequencies, especially when $f<9 \mathrm{GHz}$. In the case of the present study, the use of $12 \mathrm{GHz}$ links could be expected to be more or less sensitive to the rain microphysics. In order to assess its impact, $1 \mathrm{~min}$ drop size distributions were computed from the raindrop dataset collected by a disdrometer (Delahaye et al., 2006) over a period of 24 months between July 2008 and July 2010. Through the use of an approach similar to that proposed by Leijnse et al. (2010), for each DSD, the corresponding rain rate value $R$ and specific attenuation values $k_{f p}$ (Eq. 4) were calculated for $12 \mathrm{GHz}$ using the Mie theory (with the drops assumed to be spherical). Under these conditions, at a temperature equal to $10^{\circ} \mathrm{C}$, the specific attenuations are found to be independent of polarization. Figure 1 shows the resulting scatter plot, in which the solid line represents the power law fit. For the purposes of comparison, the dashed curve shows the power law model defined in accordance with the standard International Telecommunication Union Recommendation (ref. ITU-R, 2009). It can be seen that these two curves are very similar, and difficult to distinguish from one another. This figure shows that microphysical discrepancies can lead to an error of $\pm 7 \mathrm{~mm} \mathrm{~h}^{-1}$ in the case of rain rates greater than $10 \mathrm{~mm} \mathrm{~h}^{-1}$. The rain rate standard deviation varies from 0.2 to $6 \mathrm{~mm} \mathrm{~h}^{-1}$ when the rain rate varies from 1 to $100 \mathrm{~mm} \mathrm{~h}^{-1}$, with corresponding relative standard deviations (RSD) in the range between 20 and $6 \%$. This result shows that $12 \mathrm{GHz}$ is a non-optimal frequency for the retrieval of low rain rates, and that the proposed device would be better adapted to applications involving heavy rainfall events (flash flood forecasting, for example). The second column of Table 2 shows the coefficients obtained by computing linear regressions on the $\log (k)-\log (R)$ relationship, which are very similar to those given by the ITU model (Table 2, columns 7, 8 and 9).

Concerning the variability of the DSD along the radio link, the $k-R$ relationship can be expected to be sensitive to aggregation over domains with different volumes. Numerous studies dealing with radar measurements have investigated the $Z-R$ power law relationships (with $Z$ representing the reflectivity and $R$ the rain rate). Morin et al. (2003) have empirically shown the existence of a scale dependency of the $Z-R$ law parameters, based on the study of co-located radar and rain gauge data aggregated at different spatial scales. These authors observed a rapid increase in the parameter $a$ as a function of scale, as well as a moderate decrease in the parameter $b$. Recently, Verrier et al. $(2012,2013)$ used multifractal theory to quantify the impact of rainfall scaling properties on the Z-R relationship. These authors found that when multifractal behaviour holds simultaneously for $R$ and $Z$, the 
Table 2. Coefficients of linear regression and coefficients of determination obtained by performing $\operatorname{linear}$ regressions on $\log (k)$ and $\log (R)$ scatterplots at a frequency $f$ equal to $12 \mathrm{GHz}$ and for integration times varying between 1 and $60 \mathrm{~min}$. The last three columns indicate the ITU recommendation coefficients corresponding to horizontal, circular and vertical polarizations, respectively, at the same frequency.

\begin{tabular}{lrrrrrrrr}
\hline & $1 \mathrm{~min}$ & $5 \mathrm{~min}$ & $10 \mathrm{~min}$ & $30 \mathrm{~min}$ & $60 \mathrm{~min}$ & $\mathrm{ITU}(\mathrm{H})$ & $\mathrm{ITU}(\mathrm{C})$ & $\mathrm{ITU}(\mathrm{V})$ \\
\hline$a$ & 0.027 & 0.028 & 0.030 & 0.030 & 0.033 & 0.024 & 0.024 & 0.024 \\
$b$ & 1.15 & 1.14 & 1.13 & 1.12 & 1.1 & 1.17 & 1.15 & 1.13 \\
$R^{2}$ & 0.987 & 0.986 & 0.98 & 0.992 & 0.972 & na & na & na \\
\hline
\end{tabular}

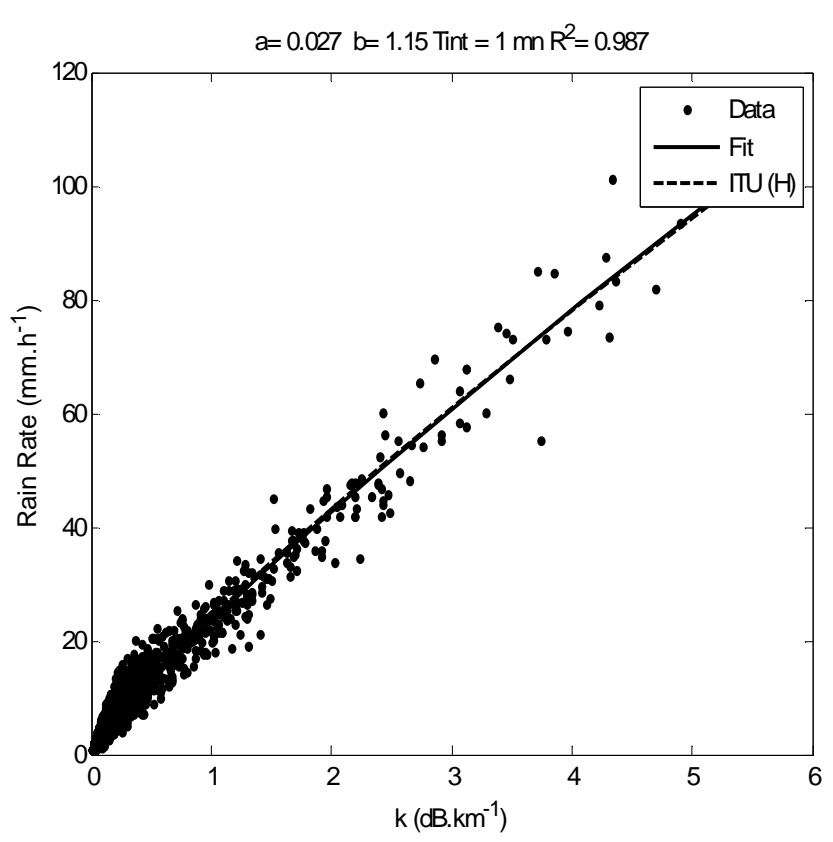

Fig. 1. Scatter plot of rain rate versus specific attenuation computed from the drop size distribution and Mie theory, for a frequency $f$ equal to $12 \mathrm{GHz}$ and a time resolution of $1 \mathrm{~min}$. The continuous line indicates the corresponding fitted $k-R$ power law. The dotted line indicates the ITU power law for the horizontal polarization.

$Z-R$ relationships can be characterized by

$\left\{\begin{array}{l}b=\text { constant } \\ a \propto l^{K_{R}(b)}\end{array}\right.$

where $K_{R}(q)$ defines the "moment scaling function" of rain of the $q$-th order, which entirely characterizes the statistics of the rain field. $K_{R}(q)$ is usually determined by the knowledge of a reduced set of "universal" parameters (Schertzer and Lovejoy, 1987). The coefficient $a$ should therefore be a power law of scale, with a scaling exponent that can be shown (from multifractal theory) to be positive when $b>1$, and negative when $b<1$. In order to quantify the impact of the scaling properties of rainfall, the study of the $Z-R$ relationship carried out by Verrier et al. $(2012,2013)$ is applied to the $k-R$ relationships in the present study. Under the assumption of a "frozen" atmosphere, an increase in integration time can be considered as equivalent to the use of larger spatial scales, and can thus allow $k-R$ relationships to be derived at different spatial scales. The raindrop dataset presented above was thus used to compute the DSD with integration times varying between 1 and $60 \mathrm{~min}$, from which the corresponding $k-R$ relationships were computed. It should be noted that Verrier et al. (2011) have shown that the rain rate series follow multifractal statistics at the mesoscale and the submesoscale. In Table 2, the stability of the resulting coefficients, associated with the high values of determination coefficient $\left(R^{2} \geq 0.972\right)$, shows that the $k-R$ relationship is rather insensitive to integration time, and thus to spatial scale (for the frequency under consideration). The robustness of the $k-R$ relationship results from the fact that the exponent $b$ is close to 1 (1.17), leading to a less critical dependence on scale of the parameters in the $k-R$ relationship than of those used in the $Z-R$ relationships, for which $b$ is close to 1.6. More specifically, in the present case, $K_{R}(1.17)$ is equal to 0.03 , whereas $K_{R}$ (1.6) is equal to 0.12 in the case of reflectivity radar. As an example, when integration times of 1 and $10 \mathrm{~min}$ are considered, these values lead to a ratio of the prefactor $a$ in the $k-R$ and $Z-R$ relationships respectively equal to 1.07 and 1.31. The latter value shows that in the case of a radar sensor, spatial-scale variability can lead to significant errors under some meteorological circumstances. Concerning the coefficient $b$, Table 2 shows that it remains almost constant (relative error $<3.5 \%$ ), as predicted by the theory. The stability of the $k-R$ relationship derived from scale properties is relatively well confirmed by the empirical values listed in Table 2, which lie in the range between 1 and approximately $30 \mathrm{~min}$, corresponding to spatial scales between a few hundred metres and a few $\mathrm{km}$, in accordance with the path length $L$ considered in this study. As the estimated $k-R$ parameters are very close to those provided by the ITU (recommendation: ITU-R, 2009), it was chosen to use these recommended values throughout the remainder of the study. Figure 2 shows the error statistics determined for specific attenuations, computed using Eq. (4), when compared to those obtained using Eq. (6) together with the ITU coefficients. A 5 min integration time was selected to represent a spatial scale compatible with the Earth-satellite path length $L$. The corresponding bias lies in the range between 1 and $6 \mathrm{~mm} \mathrm{~h}^{-1}$, with a relative error of less than $10 \%$. 


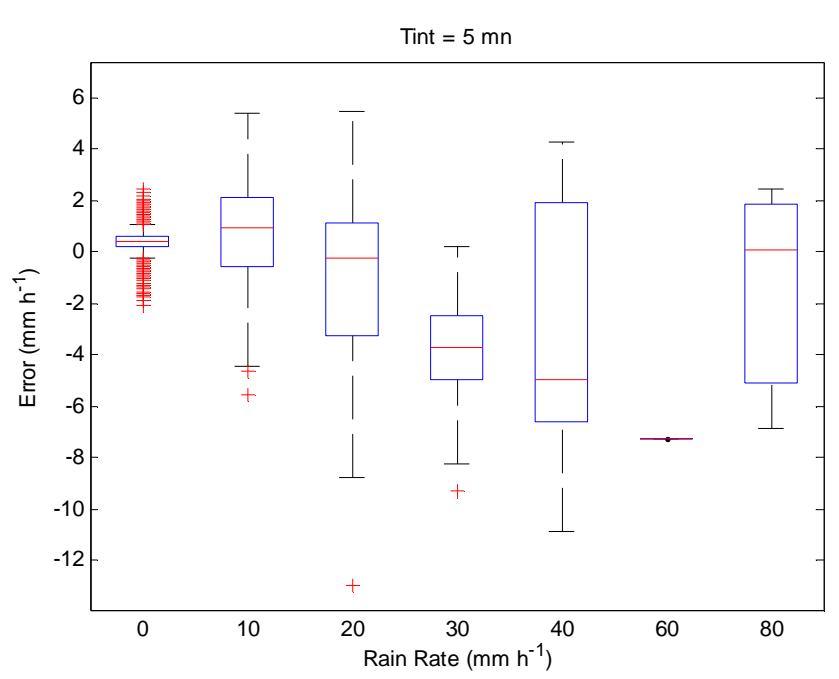

Fig. 2. Error induced on the rain estimation when the ITU power law is used for an integration time equal to $5 \mathrm{~min}$. For each rain bin, the box-and-whiskers diagram indicates the median (central vertical line), and the lower and upper quartiles (left and right edges of the box). The whiskers indicate the lower and upper limits of the distribution, within 1.5 times the interquartile range, from the lower and upper quartiles, respectively.

\section{Experimental setup}

The experimental system was installed at the LATMOS (Laboratoire Atmosphères, Milieux, Observations Spatiales) in Guyancourt, close to Paris, during the summer and autumn of 2010. The microwave signals from four geostationary satellites (NSS7, AB1, Thor 5/6, and Hot Bird 6/8/9) were received in horizontal polarization, using a $90 \mathrm{~cm}$ diameter multifocus dish antenna. The observed satellite elevations were close to $30^{\circ}$. For each satellite, two $30 \mathrm{MHz}$ wide $\mathrm{Ku}-$ band channels were received and down-converted to L-band signals by four low-noise block converters (LNBC). An RF switch allowed for sequential selection of one of the L-band signals, which was then fed to a field analyser. The latter device has an accuracy of $0.1 \mathrm{~dB}$ and is operated in spectrum mode, with a $4 \mathrm{MHz}$ bandwidth sequentially centred on each of the selected channels. The measured signal level was determined by averaging five consecutive measurements, and the averaged value was then stored in a microcomputer used for data-logging purposes. The acquisition routine sequentially records each of the eight channels, with a $2 \mathrm{~s}$ sampling period per channel, leading to a total sampling period equal to $16 \mathrm{~s}$ ( 4 satellites $\times 2$ channels $\times 2 \mathrm{~s}$ ). The experimental setup is shown in Fig. 3. The frequencies used for each link are listed in Table 3. It should be noted that in this case, the influence of water on the antenna was not taken into account, and will be discussed in Sect. 4.1.

Independent co-located rain observations were also considered in order to evaluate the performance of the proposed $\mathrm{Ku}$ device: C-band rain rate maps provided by Météo-France

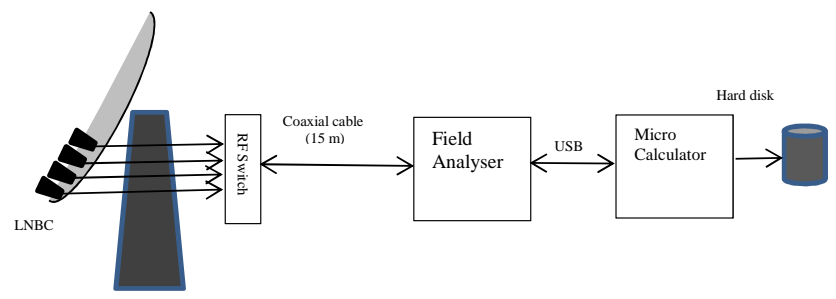

Fig. 3. Experimental setup.

weather radar located at Trappes (close to Guyancourt) and two rain gauges, also located at Trappes and Toussus le Noble, were used. The distances between the LATMOS and these locations are respectively 2.9 and $3.7 \mathrm{~km}$. Figure 4 provides a map of the various sensor locations, as well as the ground projection of the four Earth-satellite links (blue lines). The dataset can be summarized as follows:

- Eight received signal $P_{\mathrm{REC}}^{\mathrm{ch}}(t)$ time series, with "ch" ranging from 1 to 8 (4 satellites, each with 2 channels), with a 10 s sampling resolution obtained by applying a re-sampling algorithm to the original time series.

- two hourly accumulated rainfall time series, $R^{\mathrm{RG} 1}(t)$ and $R^{\mathrm{RG} 2}(t)$, obtained with the two rain gauges.

- Twenty thousand radar rain rate maps $R^{\mathrm{rad}}(t)$ with a spatial resolution of $1 \times 1 \mathrm{~km}^{2}$ and a 5 min temporal resolution. The relationship used to convert radar reflectivity factors into rain rate was identical to that used operationally by Météo-France (Tabary, 2007). The period under consideration includes most of the rainfall events which occurred between 23 July and 15 December 2010.

In this initial feasibility study, each of the eight available channels was used independently. Channel 1 was used to develop the algorithms (Sect. 4), such that seven different values of rain rate could then be estimated from the remaining channels. As these values were found to be quite similar, only those obtained with channel 7 are presented in Sect. 5.

Since microwave links provide path-averaged measurements, the corresponding average radar path rain rates are calculated from the rain maps by averaging the $R_{i}^{\mathrm{rad}}(t)$ radar pixels traversed by the link beam, and weighted by the corresponding length of the link $L_{i}$ in each pixel.

For each of the eight received channels ch, and each of the available radar maps $R^{\mathrm{rad}}(t)$, the average radar rain rate along the Earth-satellite path can be determined using the radar signals in a given channel by applying the following expression:

$\bar{R}_{\mathrm{ch}}^{\mathrm{rad}}(t)=\frac{\sum_{i \in I} L_{i} R_{i}^{\mathrm{rad}}(t)}{\sum_{i \in I} L_{i}}$,

where $R_{i}^{\text {rad }}(t)$ is the $i$-th pixel $(\mathrm{i} \in[1,262144])$ on the map, $I$ is the subset of pixels intersecting the satellite path 
Table 3. Frequency $[\mathrm{MHz}]$ of the microwave links used in the test campaign.

\begin{tabular}{cccclc}
\hline Channel & Satellite & Frequency & Channel & Satellite & Frequency $(\mathrm{MHz})$ \\
\hline 1 & NSS7 & 12604 & 5 & Thor 5/6 & 12563 \\
2 & NSS7 & 11694 & 6 & Thor 5/6 & 12688 \\
3 & AB1 & 12722 & 7 & Hot Bird 6/8/9 & 12285 \\
4 & AB1 & 12547 & 8 & Hot Bird 6/8/9 & 12617 \\
\hline
\end{tabular}

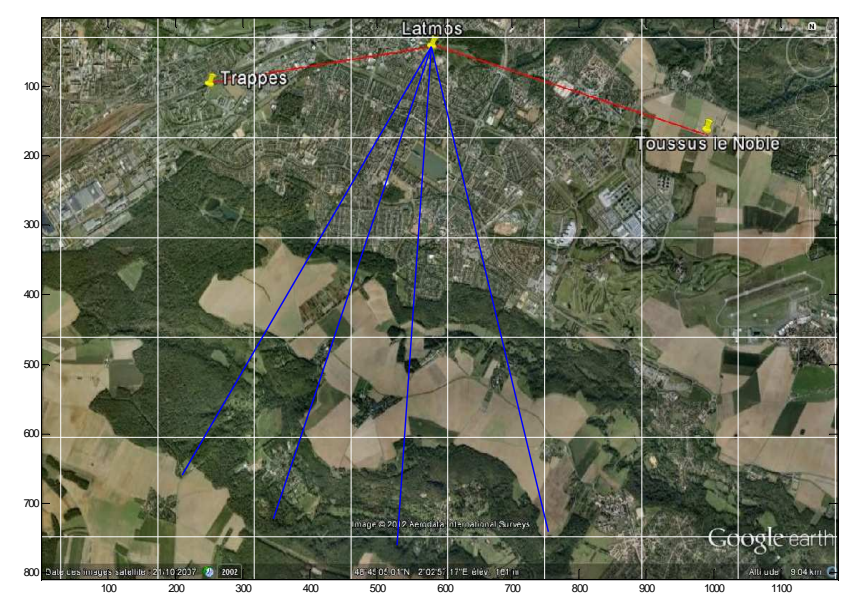

Fig. 4. Locations of the different sites and satellite path links.

corresponding to channel "ch", and $L_{i}$ is the length of the link inside the $i$-th pixel.

Finally, four time series $\bar{R}_{\mathrm{ch}}^{\mathrm{rad}}(t)$ were obtained with a $5 \mathrm{~min}$ temporal resolution, representing the path-averaged rain rates on each Earth-satellite link derived from the radar measurements.

In the case of the rain gauges, the two time series $R^{\mathrm{RG} 1}(t)$ and $R^{\mathrm{RG} 2}(t)$ were simply averaged in order to obtain $\bar{R}^{\mathrm{RG} 2}(t)$. Figure 5 shows the time series $P_{\mathrm{REC}}^{\mathrm{ch} 1}(t)$, $\bar{R}_{\mathrm{ch} 1}^{\mathrm{rad}}(t), \bar{R}_{\mathrm{RG}}(t)$ for a 10 -day period. Each rainy period is clearly characterized by a temporary fall in received signal strength, revealed by a negative pulse. The precipitation events can also be seen on the radar and rain gauge time series. It should be noted that the received signals are significantly affected by daily fluctuations $(\sim 1 \mathrm{~dB})$ resulting from apparent satellite motion and antenna aperture effects, as well as a negative trend due to a decrease in atmospheric temperature and/or water vapour content. For all of these reasons, it is very difficult to directly estimate a reference signal level, from which the rain attenuation can be derived. As explained in Sect. 4.2, correct estimation of the reference level requires dry periods to be distinguished from rainy periods, and this is achieved by analysing the observed fluctuations in signal strength.

\section{Retrieval method}

\subsection{Reference level}

The received signal strength $\left(P_{\mathrm{REC}}\right)$ expressed by Eq. (8) combines the parameters related to instrumental and geometric characteristics, such as the satellite transmitter power $\left(P_{\mathrm{E}}\right)$, the transmitter and receiver antenna gains $G_{\mathrm{E}}, G_{\mathrm{R}}$, the free-space attenuation $A_{\mathrm{F}}$ and the tropospheric attenuation $\left(A^{\text {Trop }}\right)$. Wet antennae can introduce additional attenuation effects, depending on the type of antenna (Schleiss et al., 2013; Crane, 2002; Leijnse et al., 2008). To minimize such effects, a super hydrophobic coating was applied to the dish and the horn. This type of coating is efficient, and even though it does not completely eliminate the presence of all raindrops, the attenuation produced by residual raindrops on the ground antenna is sufficiently small to be neglected, and thus does not appear in Eq. (8). Moreover in the case of Earth-space microwave links, only the ground antenna can become wet.

$P_{\mathrm{REC}}(t)=P_{\mathrm{E}}(t)+G_{\mathrm{E}}(t)+G_{\mathrm{R}}(t)-A_{\mathrm{F}}(t)-A^{\mathrm{Trop}}(t)(\mathrm{dB})$

In practice, $P_{\mathrm{E}}$ and $G_{\mathrm{E}}$ remain almost constant, whereas $G_{\mathrm{R}}$ and $A_{\mathrm{F}}$ vary slowly over time due to apparent satellite motion. The tropospheric attenuation $A^{\text {Trop }}$ is critically dependent on satellite elevation and the frequency band under consideration. In the Ku band, although oxygen ( $\left.A^{\text {Oxygen }}\right)$, liquid water in clouds ( $\left.A^{\text {Cloud }}\right)$, water vapour $\left(A^{\text {vapour }}\right)$ and scintillation $\left(A_{\mathrm{S}}\right)$ have an influence on signal strength, rain $\left(A^{\text {Rain }}\right)$ is the dominant contributor to the overall attenuation.

$$
\begin{gathered}
A^{\text {Trop }}(t)=A^{\mathrm{S}}(t)+A^{\text {Oxygen }}(t)+A^{\text {Cloud }}(t) \\
+A^{\text {Vapour }}(t)+A^{\text {Rain }}(t) \quad(\mathrm{dB})
\end{gathered}
$$

For the purposes of estimating the rain attenuation $\left(A^{\text {Rain }}\right)$, Eq. (8) is expressed as follows:

$P_{\mathrm{REC}}(t)=P_{\mathrm{REF}}(t)-A^{\mathrm{Rain}}(t) \quad(\mathrm{dB})$,

where $P_{\operatorname{PREF}}(t)$, which is called the reference level or baseline, is given by

$$
\begin{aligned}
& P_{\mathrm{REF}}(t)=P_{\mathrm{E}}+G_{\mathrm{E}}+G_{\mathrm{R}}-A_{\mathrm{F}}-A^{\mathrm{S}}(t) \\
& \quad-A^{\text {Oxygen }}(t)-A^{\text {Cloud }}(t)-A^{\text {Vapour }}(t) \quad(\mathrm{dB}) .
\end{aligned}
$$

In the absence of rainfall, we have

$P_{\mathrm{REC}}(t)=P_{\mathrm{REF}}(t) \quad(\mathrm{dB})$. 


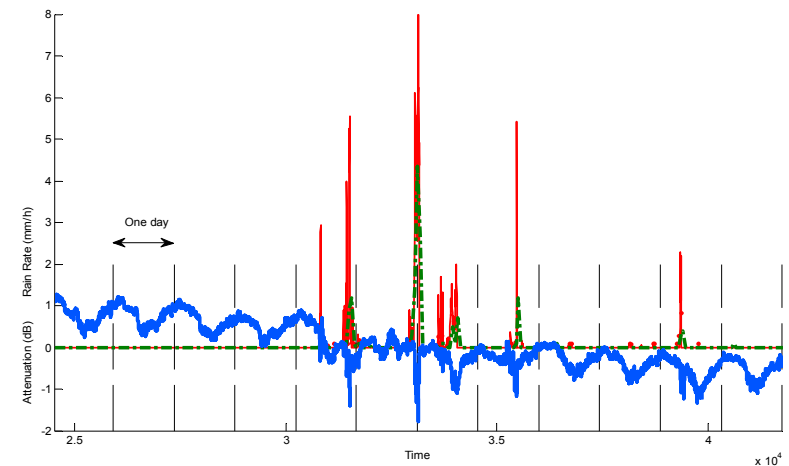

Fig. 5. Example of recorded time series: received signal (blue curve), radar rain rate (red curve) and $1 \mathrm{~h}$ accumulated rainfall time series (dashed green curve).

As the reference level is observed directly during non-rain situations, during rainfall events its value must be estimated from the reference level obtained during dry periods (Fig. 6), and it is thus essential to develop an algorithm allowing for dry periods to be differentiated from rainy periods. Once the reference level is known, Eqs. (5), (6) and (9) allow for the corresponding rain rate $\bar{R}_{\mathrm{ch}}^{\mathrm{KU}}(t)$ to be estimated.

\subsection{The rain-no-rain detection algorithm}

Each of the atmospheric processes involved has its own dynamics, and contributes differently to the attenuation of the propagating electromagnetic wave. As a consequence of its strong heterogeneity, rain leads to much more rapid temporal fluctuations of the received signal than gases or clouds. In order to distinguish between dry and rainy periods, an approach similar to those described by Kaufmann and Rieckermann (2011) and Schleiss and Berne (2010) is proposed. The various characteristics (trends, standard deviation, kurtosis, skewness) of the observed Ku-band signal received on channel 1 were computed using different window sizes centred on current time, and ranging from $100 \mathrm{~s}$ to $1 \mathrm{~h}$. The radar data were used to determine the corresponding state of the atmosphere (rainy or dry). The statistical distributions of these rainy and dry period characteristics were compared in order to test their ability to discriminate between these two states. The selected window width $W$ should not be too large, since it determines the time delay needed to obtain the estimate. Since the aim of this study is to develop a sensor allowing for near-real-time observations to be made, the time windows were chosen to be as small as possible, whilst ensuring good discrimination.

Two characteristics were selected (see Eq. 10): the standard deviation for a $30 \mathrm{~min}$ time window, and the local trend

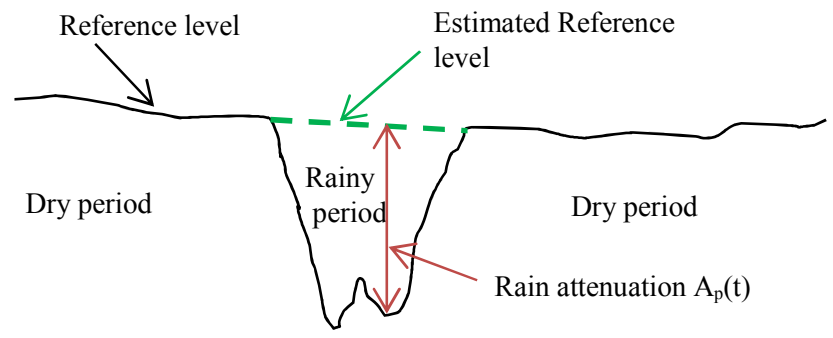

Fig. 6. Received signal during a rain event.

over a 4 min time window.

$$
\left\{\begin{array}{l}
\operatorname{std}\left(P_{\mathrm{REC}}(n)\right)=\left[\frac{1}{2 W+1} \sum_{i=-L}^{L}\left(P_{\mathrm{REC}}(n+i)-\bar{P}_{\mathrm{REC}}(n)\right)^{2}\right] \frac{1}{2} \\
\text { with } \bar{P}_{\mathrm{REC}}(n)=\frac{1}{2 W+1} \sum_{i=-L}^{L} P_{\mathrm{REC}}(n+i) \text { and } W=100 \\
\operatorname{Trd}\left(P_{\mathrm{REC}}(n)\right)=\frac{1}{W} \sum_{i=-L}^{L} a_{i} P_{\mathrm{REC}}(n+i) \\
\text { with } a=(-1,-1, \ldots-1,0,1, \ldots 1) \text { and } W=10
\end{array}\right.
$$

The aim of this approach was to develop a pattern classifier, providing an appropriate rule for the assignment of each sample $P_{\text {REC }}(n)$ into one of the two classes (rainy or dry). Since the optimal boundary between the two regions, referred to as a decision boundary, is non-linear, it was considered preferable to make use of an artificial neural network. The so-called multi-layer perceptron (MLP) algorithm used here, which is able to learn complex (non-linear) and multi-dimensional mapping from a collection of examples, is an ideal classifier (Haykin, 1999); the MLP is defined by its topology, namely the dimensions of the input and output vectors and the number of hidden neurons, and by its weights. A training process is needed to determine the optimal weights. This step is called the training process, and requires a representative database comprising a wide set of input and output vectors $\left(X^{n}, Y^{n}\right)$ :

$$
\left\{\begin{aligned}
X^{n} & =\left[\operatorname{std}\left(P_{\mathrm{REC}}(n)\right), \operatorname{Trd}\left(P_{\mathrm{REC}}(n)\right)\right] \\
Y^{n} & =\left\{\begin{array}{c}
0 \text { if } \bar{R}_{\mathrm{ch} 1}^{\mathrm{rad}}(n)<0.1 \mathrm{mmh}^{-1} \\
\text { belongs to a dry period } \\
1 \text { if } \bar{R}_{\mathrm{ch} 1}^{\mathrm{rad}}(n)>0.1 \mathrm{mmh}^{-1} \\
\text { belongs to a rainy period. }
\end{array}\right.
\end{aligned}\right.
$$

The training database comprises half of the dataset measured by channel 1 (1336201 samples), whereas the other half is used to determine the optimal architecture (six hidden neurons) in terms of generalization ability. The generalization property makes it possible to train an MLP with a representative set of input/target pairs, and to obtain good results when predicting unseen input samples. Following training, the MLP output provides a direct estimation of the posterior probabilities (Zhang, 2000), as shown in Fig. 7a.

Once it has been determined, the MLP can be applied to the entire dataset for the purposes of identifying dry and rainy 

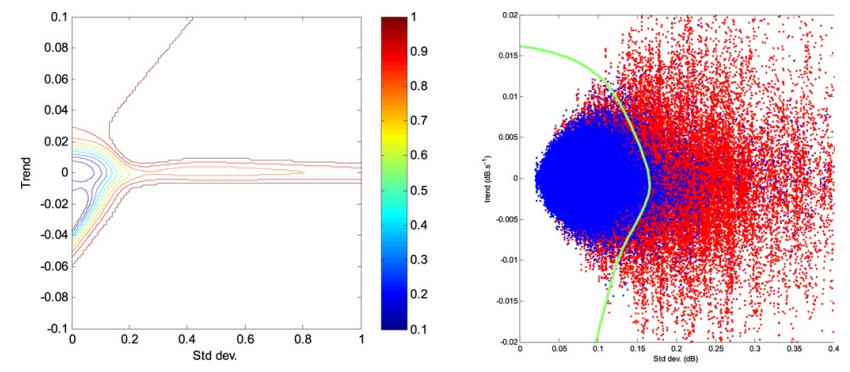

Fig. 7. (a) MLP output: posterior rain class probability. (b) Boundary of the classifier (green solid line), samples corresponding to the dry class are in blue, and samples corresponding to the rainy class are in red.

episodes. The discriminating rule is simple: assign sample $n$ to the rainy class if $Y^{n} \geq P_{\mathrm{o}}$, or to the dry class if $Y^{n}<P_{\mathrm{o}}$. Figure $7 \mathrm{~b}$ provides a plot of the boundary (solid line) between the two classes. The determination of $P_{\mathrm{o}}$ is described in detail below.

Following the identification of dry and rainy periods based on MLP analysis of the received signals, the reference level is interpolated during rainy periods, allowing for the rain attenuation $A^{\text {Rain }}(t)$ to be estimated. Using Eq. (12) to express the geometric path length $L$ (ITU-R, 2009), the specific attenuation $k_{f p \theta}(t)$ can be estimated using Eq. (5).

$$
\begin{aligned}
& L=\frac{h_{\mathrm{R}}-h_{\mathrm{S}}}{\sin (\theta)} \\
& h_{\mathrm{R}}=h_{0}+0.36(\mathrm{~km}),
\end{aligned}
$$

where $h_{\mathrm{R}}$ is the altitude of the top of the layer of rain and $h_{0}$ is the annual average altitude above mean sea level of the $0^{\circ} \mathrm{C}$ isotherm. According to the ITU-R (2001) recommendation, the latter parameter can be taken to be $3 \mathrm{~km}$ in the present case. The parameter $h_{\mathrm{S}}$ is the altitude of the ground station and $\theta$ is the elevation angle. Finally, the corresponding rain rate $\bar{R}_{\mathrm{ch} 1}^{\mathrm{KU}}(t)$ is given by Eq. (6). The probability threshold $P_{\mathrm{o}}$ (determined as 0.55 ) is chosen so that the CDF corresponding to the $1 \mathrm{~h}$ accumulated $\bar{R}_{\mathrm{ch} 1}^{\mathrm{KU}}(t)$ is as close as possible to that of $\bar{R}_{\mathrm{ch} 1}^{\mathrm{KU}}(t)$ (see Fig. 8). It should be noted that this threshold gives a percentage of rain similar to that obtained over the same period with a disdrometer, located $10 \mathrm{~km}$ from the receiver, when a $0.1 \mathrm{~mm} \mathrm{~h}^{-1}$ threshold is applied.

\section{Validation and results}

The method described in the preceding sections was used to estimate $\bar{R}_{\mathrm{ch} 7}^{\mathrm{KU}}(t)$. A characteristics vector $\boldsymbol{X}$, corresponding to channel 7, was computed from the time series $P_{\mathrm{REC}}^{\text {ch77 }}(t)$ and then applied to the MLP, with which the threshold $\mathrm{P}_{o}$ was used to discriminate between rainy and dry periods. In order to quantify the performance of the device and associated algorithm, comparisons were then made with $\bar{R}_{\mathrm{ch} 7}^{\mathrm{rad}}(t)$ and $\bar{R}^{\mathrm{RG}}(t)$ using different criteria. Firstly, the rainfall inten-

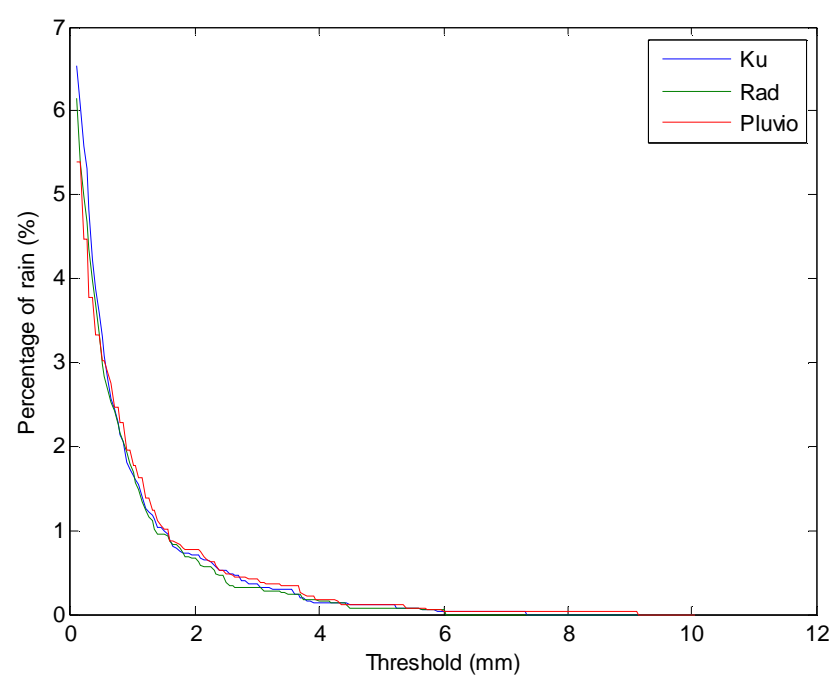

Fig. 8. Percentage of rain vs. $1 \mathrm{~h}$ accumulated rain threshold for the radar time series (green), rain gauges (red) and KU device (blue) for a threshold $P_{\mathrm{O}}=0.55$.

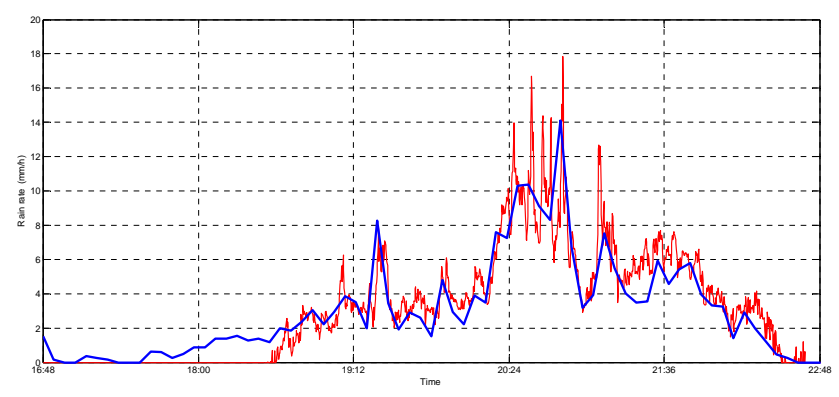

Fig. 9. Examples of time series recorded by the $\mathrm{Ku}$ device (red curve) and the radar (blue curve).

sity time series measured by the rain radar and determined with the $\mathrm{Ku}$ device were compared visually. The order of magnitude and dynamics of the two measurements can be seen to be similar (Fig. 9), even though these two quantities cannot be rigorously equal as a consequence of the different sampling volumes, altitudes and time resolutions involved in these two sets of data. Figure 10 compares the accumulated rainfall recorded by the gauges and rain radar, with the values estimated from the received signals over a 4 month period. The same accumulated rainfall, i.e. a total height of $220 \mathrm{~mm}$, is determined by the radar and the Ku device, whereas the rain gauges record a slightly higher value.

Figure 11 provides a comparison between the hourly accumulated rainfall measured by the radar and the Ku device (top), and between the rain gauge and the $\mathrm{Ku}$ device (bottom). The quantile-quantile plots (left-hand side of the figure) are relatively close to the diagonal, showing that the $1 \mathrm{~h}$ accumulated rainfall distributions are quite similar. Error box plots are provided on the right-hand side of this figure. The median values are close to zero, especially in the case of the 


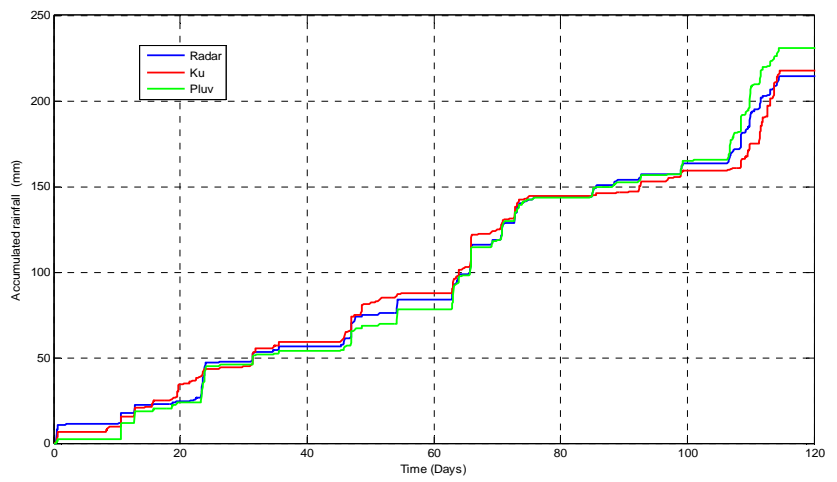

Fig. 10. Accumulated rainfall determined using the radar data (blue curve), rain gauge (green curve) and $\mathrm{Ku}$-band attenuation measurements (red curve) over a 4 month period.

comparison between the $\mathrm{Ku}$ device and the radar, with the exception of the intermediate value of accumulated rainfall $(-1.3 \mathrm{~mm})$. In the case of both box plots, it can be seen that the width of the boxes, defined as the separation between the 25th and 75th percentiles, increases when the accumulated rainfall increases. Several explanations are proposed for this phenomenon in the following paragraph.

\section{Rainfall event case studies}

Eight rainfall events of various intensities and durations were selected. Although these cannot be considered to be representative of the local rainfall climatology, they nonetheless make it possible to highlight several features of the Ku-band rainfall data. The dates, durations, maximum rain rates and quantities of rain estimated by the radar, the rain gauges and the $\mathrm{Ku}$ device are provided in Table 4 . The events are sorted by increasing duration. Note that the maximum rain rates $R_{\max }$ are obtained with different integration times, depending on the type of measurement (rain gauges: $1 \mathrm{~h}$, rain radar: $5 \mathrm{~min}$, Ku device $1 \mathrm{~s}$ ). It can be seen that the quantities of rain estimated by the radar and the Ku-band sensor are relatively close to each other (deviation $<15 \%$ ) for events lasting between 1 and 3 hours (events 3, 4, 5 and 6). However, this is not the case for very brief $(<1 \mathrm{~h})$ events, nor for long rainfall events $(>6 \mathrm{~h})$. In the case of the short-duration events (events 1 and 2), the maximum values of $R_{\max }$ for the rain radar and $\mathrm{Ku}$ device are relatively close to each other, although it is difficult to compare the measured quantities of rain as a consequence of the small size of the rain cells. Indeed, in the case of small rain cells traversed more or less perpendicularly by the microwave link, the cell is observed for only a few seconds or minutes, whereas it is observed for a longer period of time in the corresponding rain radar pixel, since the latter are much greater in size $(1 \mathrm{~km})$ than those corresponding to the microwave link (a few metres). Thus, the two instruments may not necessarily have observed exactly
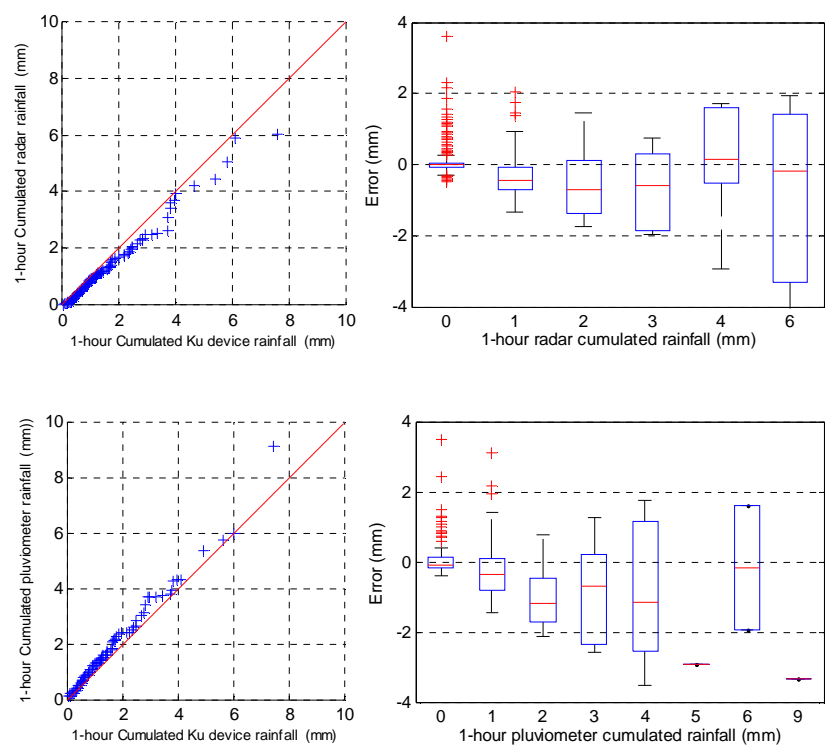

Fig. 11. Q-Q plots using radar and $\mathrm{Ku}$ device data (top left), and rain gauge and $\mathrm{Ku}$ device data (bottom left). Box plots made using radar and $\mathrm{Ku}$ device data (top right), and rain gauge and $\mathrm{Ku}$ device data (bottom right).

the same phenomena. In the case of long-duration rainfall events (events 7 and 8), the reason for the discrepancy in the observations is completely different. The simple interpolation technique used to calculate the reference level appears to have been unsuitable because of daily variations of reference level, and a more sophisticated method should be used in the future. It should be noted that in all cases, there are several reasons for which it is difficult to compare the results obtained with these two devices: (i) it is well known that the radar $Z-R$ equation sometimes overestimates or underestimates the rain rate, (ii) the path length $L$ can be underor over-estimated if the $0^{\circ}$ isotherm is not sufficiently well known, and (iii) in the presence of high rainfall rates, associated with very strong spatial heterogeneities, significant differences can occur because the instruments do not see the same volume of the atmosphere.

\section{Conclusions}

Ku-band microwave sources on geostationary satellites, such as those used in telecommunications or broadcasting, can potentially be used for the estimation of rainfall. A low-cost, ground-based microwave system allowing for atmospheric attenuation to be estimated along Earth-satellite links was developed in order to investigate the opportunistic use of these microwave sources in the frequency band between 10.7 and $12.7 \mathrm{GHz}$. Although this band is not optimal for the estimation of weak rainfall rates, due to its lack of sensitivity, it appears to be a good choice when the rainfall rate increases, since in this case the attenuation rarely exceeds $12 \mathrm{~dB}$, even 
Table 4. Features of the eight selected events.

\begin{tabular}{clccccccc}
\hline Ev. \# & Date & $\begin{array}{c}\text { Duration } \\
(\mathrm{h})\end{array}$ & $\begin{array}{c}R_{\max } \operatorname{Rad} \\
\left(\mathrm{mm} \mathrm{h}^{-1}\right)\end{array}$ & $\begin{array}{c}R_{\max } \text { Pluv } \\
\left(\mathrm{mm} \mathrm{h}^{-1}\right)\end{array}$ & $\begin{array}{c}R_{\max } \mathrm{Ku} \\
\left(\mathrm{mm} \mathrm{h}^{-1}\right)\end{array}$ & $\begin{array}{c}\text { Amount } \\
\operatorname{Rad}(\mathrm{mm})\end{array}$ & $\begin{array}{c}\text { Amount } \\
\text { Pluv }(\mathrm{mm})\end{array}$ & $\begin{array}{c}\text { Amount } \\
\mathrm{Ku}(\mathrm{mm})\end{array}$ \\
\hline 1 & 23 July & 0.6 & 25.8 & - & 22.1 & 5.8 & - & 3 \\
2 & 16 October & 0.8 & 7.3 & - & 8.3 & 1.7 & - & 0.9 \\
3 & 03 October & 1.5 & 14.4 & 1.6 & 20.5 & 4.9 & 1.6 & 5.6 \\
4 & 04 August & 1.65 & 30.3 & 0.9 & 28.4 & 5.4 & 1 & 4.8 \\
5 & 02 August & 1.7 & 19.7 & 9.1 & 40 & 5.7 & 9.1 & 6.7 \\
6 & 07 September & 2.9 & 7.9 & 4.3 & 8.1 & 8.3 & 7 & 7.8 \\
7 & 23 August & 6.0 & 8.4 & 3.7 & 16.6 & 4.6 & 5.4 & 7.6 \\
8 & 08 November & 9.2 & 12.5 & 4 & 7.1 & 12.2 & 10.3 & 5.4 \\
\hline
\end{tabular}

in the presence of very heavy rainfall, and the signals can be measured without difficulty even with a parabolic antenna of standard dimensions $(90 \mathrm{~cm})$. Nevertheless the use of $\mathrm{Ku}-$ band microwave sources could be very useful in the context of risk management (flash flood) or urban hydrology, for which high rain rates have more importance. Finally, because of their spatial resolution close to that of radar, we could also consider their use for calibration of ground or space precipitation radar.

A four month measurement campaign was carried out, following which a multi-layer neural network was used to distinguish between dry and rainy periods, on the basis of the received signal, thus allowing for the attenuation produced by rain along the path of the $\mathrm{Ku}$-band link to be estimated. ITU models were then used to convert rain attenuation into a rainfall rate. Good agreement is found between the values determined with the $\mathrm{Ku}$ device, weather radar and rain gauges, not only in terms of total accumulated rainfall but also in terms of hourly rainfall distributions. In the case of $1 \mathrm{~h}$ accumulated rainfall, discrepancies of as much as $30 \%$ can occur between the radar and $\mathrm{Ku}$ device observations. This is explained by the large differences in sample volume and integration time used with these two devices, which do not "see" exactly the same parts of the atmosphere. These differences can become particularly significant during heavy rainfall events, which are generally associated with very strong spatial heterogeneities. Although the results presented here provide validation of the proposed technique which is only partial, and despite the simplicity of some of the models and the use of empirical formulas, these initial results are promising. Since the parameter $b$ of the $k-R$ law is close to 1 , the variability of the DSD along the Ku-band link does not play an important role. Nevertheless, some variations in attenuation, not induced by rain, may occur, as in the case of heavy rain events, which are known to exhibit complex spatial and temporal structures with strong temperature gradients and a complex vertical humidity profile. Moreover, in this study, the possible presence of hail, wet snow or a melting layer is not taken into account. An attenuation of approximately $0.2-0.3 \mathrm{~dB}$ can be expected in the $\mathrm{Ku}$ band when a melting layer is present, i.e. mainly during stratiform events. However, when non-precipitating clouds with a melting layer are present just before a rainfall event, the resulting attenuation (due to the clouds and the melting layer) is "embedded" into the reference level estimation, and consequently does not lead to a strong error in the rain attenuation estimation. It is difficult to compare the results presented here with those obtained by Maitra et al. (2007), who compared rain attenuation with values determined with a co-localized disdrometer and a simple attenuation model (SAM) (Stutzman and Yon, 1986). These authors conclude that "the discrepancy becomes significant when the rain rates are large (above $30 \mathrm{~mm} \mathrm{~h}^{-1}$ ) and the corresponding rain cell size is small". They explain that, in the case of high rain rates, the rain rate decay parameter used in the SAM is no longer valid, and that as a consequence no meaningful conclusions can be drawn.

Several possibilities could be investigated to improve the performance of the technique proposed in the present study, during which each of the available channels (i.e. one frequency and one polarization) was used independently to estimate different values of rain rate. In future test campaigns, the combined use of several channels, corresponding to different frequencies or polarizations, should improve the global performance of this technique. In the case of longduration rain events $(>3 \mathrm{~h})$, improvements are still needed, especially for the evaluation of the reference level of attenuation. Indeed, in the present study, a simple linear interpolation of dry period data was used. This approach is sufficient for short periods only, during which the reference level is affected by small variations. For observations made over longer periods, a more sophisticated estimation of the reference level is needed. As an example, this could be achieved by representing the reference level by a state-space model associated with a Kalman filter. ITU models were used for the $k-R$ relationship and to estimate the $\mathrm{Ku}$-band path length. Although the results of this study show that the ITU $k-R$ relationship is perfectly suited to this problem, this may not be the case when it comes to the path length estimation. Other models have been described, such as that proposed by Adhikari et al. (2011), and these should also be tested. 
The present study deals with the estimation of rainfall rate, using Ku-band attenuation over a single path link. In the future, the use of several simultaneous links associated with tomography or assimilation methods, based on an approach similar to that of Zinevich et al. $(2008,2009)$ or Giulli et al. (1997, 1999), could be applied to the estimation of small-scale rainfall fields, and could be helpful in hydrological applications, flash flood forecasting and weather radar calibration.

Acknowledgements. This work was supported by the French "Programme National de Télédétection Spatiale" (PNTS, http://www.insu.cnrs.fr/actions-sur-projets/ pnts-programme-national-de-teledetection-spatiale), grant no. PNTS-2013-01. The authors would like to thank M. Parent du Chatelet from Météo-France for various fruitful discussions on the topic of radar data processing.

Edited by: F. S. Marzano

\section{References}

Adhikari, A., Das, S., Bhattacharya, A., and Maitra, A.: Improving rain attenuation estimation: modelling of effective path length using ku-band measurements at a tropical location, Prog. Electromagn. Res. B, 34, 173-186, 2011.

Atlas, D. and Ulbrich, C. W.: Path- and Area-Integrated Rainfall Measurement by Microwave Attenuation in the 1-3 cm Band, J. Appl. Meteor., 16, 1322-1331, 1977.

Berne, A. and Uijlenhoet, R.: Path-averaged rainfall estimation using microwave links: Uncertainty due to spatial rainfall variability, Geophys. Res. Lett., 34, L07403, doi:10.1029/2007GL029409, 2007.

Crane, R. K.: Analysis of the effects of water on the ACTS propagation terminal antenna, Antennas and Propagation, IEEE Trans., 50, 954-965, 2002.

Delahaye, J. Y., Barthès, L., Golé, P., Lavergnat, J., and Vinson, J. P.: a dual beam spectropluviometer concept, J. Hydrol., 328, 110-120, 2006.

Fenicia, F., Pfister, L., Kavetski,D., Matgen, P., Iffly, J. F., Hoffmann, L., and Uijlenhoet, R.: Microwave links for rainfall estimation in an urban environment: Insights from an experimental setup in Luxembourg-City, J. Hydrol., 464-465, 69-78, 2012.

Giuli, D., Facheris, L., and Tanelli, S.: A new microwave tomography approach for rainfall monitoring over limited areas, Phys. Chem. Earth, 22, 265-273, 1997.

Giuli, D., Facheris, L., and Tanelli, S.: Microwave tomographic inversion technique based on stochastic approach for rainfall fields monitoring, IEEE T. Geosci. Remote, 37, 2536-2555, 1999.

Haykin, S.: Neural Networks: comprehensive Foundation, PrenticeHall, Upper Saddle River, N.J., 1999.

ITU-R: Propagation data and prediction methods required for the design of terrestrial line-of-sight systems: Recommendation ITU-R P.530 -9, 2001.
ITU-R: Propagation data and prediction methods required for earthspace telecommunication systems: Recommendation ITUR P.618-9, ITU-R Recommendations, P-Series Fascicle, ITU, Geneva, 2009.

ITU-R: Attenuation by atmospheric gases, Recommendation ITUR P.676-9, available at: www.itu.int/rec/R-REC-P.676 (last access: 28 August 2013), 2012a.

ITU-R: Attenuation due to clouds and fog, Recommendation ITUR P.840-5, available at: www.itu.int/rec/R-REC-P.840 (last access: 28 August 2013), 2012b.

Jameson, A. R.: A Comparison of Microwave Techniques for Measuring Rainfall. J. Appl. Meteor., 30, 32-54, 1991.

Kaufmann, M. and Rieckermann, J.: Identification of dry and rainy periods using telecommunication microwave links, 12nd International Conference on Urban Drainage, Porto Alegre/Brazil, 10-15 September, 2011, available at: http://www.yumpu.com/en/document/view/6346597/ identification-of-dry-and-rainy-periods-using-telecommunication-, 2011.

Kumar, S., Bhaskara, V., and Narayana Rao, D.: Prediction of Ku Band Rain Attenuation Using Experimental Data and Simulations for Hassan, India, Int. J. Comput. Sci. Netw. Sec., 8, 10-15, 2008.

Leijnse, H., Uijlenhoet, R., and Stricker, J. N. M.: Rainfall measurement using radio links from cellular communication networks, Water Resour. Res., 43, W03201, doi:10.1029/2006WR005631, 2007.

Leijnse, H., Uijlenhoet, R., and Stricker, J. N. M.: Microwave link rainfall estimation: Effects of link length and frequency, temporal sampling, power resolution, and wet antenna attenuation, Adv. Water Resour., 31, 1481-1493, 2008.

Leijnse, H., Uijlenhoet, R., and Berne, A.: Errors and Uncertainties in Microwave Link Rainfall Estimation Explored Using Drop Size Measurements and High-Resolution Radar Data, J. Hydrometeor., 11, 1330-1344, 2010.

Liebe, H. J., Hufford, G. A., and Cotton, M. G.: Propagation Modeling of Moist Air and Suspended Water/Ice Particles at Frequencies below 1000 GHz, AGARD Conference, Atmospheric Propagation Effects through Natural and Man-Made Obscurants for Visible to MM-Wave Radiation, 542, 3.1-3.10, 1993.

Maitra, A., Kaustav, C., Sheershendu, B., and Srijibendu, B.: Propagation studies at Ku-band over an earth-space path at Kolkata, Ind. J. Radio Sp. Phys., 36, 363-368, 2007.

Messer, H., Zinevich, A., and Alpert, P.: Environmental monitoring by wireless communication networks, Science, 312, 713-716, 2006.

Morin, E., Krajewski, W. F., Goodrich, D., Xiaogang, G., and Sorooshian S.: Estimating rainfall intensities from weather radar data: the scale-dependency problem, J. Hydromet., 4, 782-797, 2003.

Olsen, R. L., Rogers, D. V., and Hodge, D. B .: The aRb relation in the calculation of the rain attenuation, IEEE Trans. Antennas Propag., 26, 318-329, 1978.

Overeem, A., Leijnse, H., and Uijlenhoet, R.: Country-wide rainfall maps from cellular communication networks, Proc. Natl. Acad. Sci., 110, 2741-2745, doi:10.1073/pnas.1217961110, 2013.

Ramachandran, V. and Kumar, V.: Rain Attenuation Measurement on Ku-band Satellite TV Downlink in Small Island, Electron. Lett., 40, 49-50, 2004. 
Schertzer, D. and Lovejoy, S.: Physically based rain and cloud modeling by anisotropic, multiplicative turbulent cascades, J. Geophys. Res., 92, 9692-9714, 1987.

Schleiss, M. and Berne, A.: Identification of dry and rainy periods using telecommunciation microwave links, IEEE Geosci. Remote Sens. Lett., 7, 611-615, 2010.

Schleiss, M., Rieckermann, J., and Berne, A.: Quantification and modeling of wet-antenna attenuation for commercial microwave links, IEEE Geosci. Remote Sens. Lett., PP (99), doi:10.1109/LGRS.2012.2236074, 2013.

Stutzman, W. L. and Yon, K. M.: A simple rain attenuation model for earth-space radio links operating at 10-35 GHz, Radio Sci., 21, 65-72, doi:10.1029/RS021i001p00065, 1986.

Tabary, P.: The new french operational radar rainfall product. Part I: methodology, Weather Forecast., 22, 393-408, 2007.

Ulaby, F. T., Moore, R. K., and Fung, A. K.: Microwave Remote Sensing, Active and Passive, Vol. 1, Microwave Remote Sensing Fundamentals and Radiometry, Artech house Inc., 1981.

Upton, G. J. G., Holt, A. R., Cummings, R. J., Rahimi, A. R., and Goddard, J. W. F.: microwave links: the future for urban rainfall measurement?, Atmos. Res., 77, 300-312, 2005.

Verrier, S., Mallet, C., and Barthès, L.: Multiscaling properties of rain in the time domain, taking into account rain support biases, J. Geophys. Res., 116, D20119, doi:10.1029/2011JD015719, 2011.
Verrier, S., Barthes, L., and Mallet, C.: Scaling properties of rainfall in space and time and their impact on rain rate estimation from weather radar 7th European Conference on Radar in Meteorology and Hydrology ERAD2012, Toulouse France, 2012.

Verrier, S., Barthes L., and Mallet C.: Theoretical and empirical scale-dependency of Z-R relationships: evidence, impacts and correction, J. Geophys. Res., 118, 7435-7449, doi:10.1002/jgrd.v118.14, 2013.

Wang, Z., Schleiss, M., Jaffrain, J., Berne, A., and Rieckermann, J.: Using Markov switching models to infer dry and rainy periods from telecommunication microwave link signals, Atmos. Meas. Tech., 5, 1847-1859, doi:10.5194/amt-5-1847-2012, 2012.

Zhang, G. P.: Neural Networks for Classification: A Survey, IEEE Trans. Syst., Man, Cybern., Part C: applications and reviews, 30, 451-462, 2000.

Zinevich, A., Alpert, P., and Messer, H.: Estimation of rainfall fields using commercial microwave communication networks of variable density, A. Water Res., 31, 1470-1480, 2008.

Zinevich, A., Messer, H., and Alpert, P.: Frontal Rainfall Observation by a Commercial Microwave Communication Network, J. Appl. Meteor. Climatol., 48, 1317-1334, 2009. 